\title{
Gefäßbildgebung zur Kontrolle bei Entzündung großer Gefäße
}

Banerjee S et al. Effect of Treatment on Imaging, Clinical, and Serologic Assessments of Disease Activity in Large-vessel Vasculitis. J Rheumatol 2020; 47: 99-107

Die Krankheitsaktivität einer Entzündung großer Gefäße (LVV) wird traditionell eher anhand klinischer und serologischer Variablen als anhand der Gefäßbildgebung beurteilt. In einer Studie wurde die Wirkung der Behandlung mittels 18F-Fluordesoxyglucose-Positronenemissionstomografie (FDG-PET) gemessen und klinische, serologische und bildgebungsbasierte Bewertungen miteinander verglichen.

Für ihre prospektive Studie schlossen die US-amerikanischen Wissenschaftler Patienten mit Riesenzell-Arteriitis (GCA) oder Takayasu-Arteriitis (TA) ein, die in Intervallen von 6 Monaten untersucht wurden. Bei jedem Untersuchungstermin erfolgten klinische Evaluationen, Bildgebung und Labor- 
untersuchungen. Basierend auf der klinischen Untersuchung erfolgte eine Beurteilung des Erkrankungszustands anhand eines Scores von 0-10 (Physician's Global Assessment, PGA).

Behandlungsänderungen wurden mindestens 3 Monate vor dem Kontrollbesuch vorgenommen und als erhöht, verringert oder unverändert eingestuft. Die bildgebende (qualitative FDG-PET-Analyse), klinische und serologische (Blutsenkung, C-reaktives Protein) Ergebnisse bei jedem Untersuchungstermin wurden miteinander verglichen.

Die Studienpopulation bestand aus $52 \mathrm{~Pa}$ tientinnen und Patienten, davon 31 mit GCA und 21 mit TA. Der Anteil weiblicher Patienten betrug 75\%. Das mediane Alter der TA-Patientinnen und Patienten lag bei 30 (20-37) Jahren, das der GCA-Patientinnen und Patienten war signifikant höher (72 [62-76] Jahre).

Die mediale Erkrankungsdauer der Patientinnen und Patienten mit GCA zu Studienbeginn war signifikant kürzer, als das der mit TA (1,4 vs. 4,8 Jahre). Die Patientinnen und Patienten mit GCA nahmen in $42 \%$ der Fälle DMARDs und/oder Biologika ein und in $68 \%$ der Fälle Kortikosteroide.

Die meisten Patientinnen und Patienten mit TA (81\%) nahmen DMARDs und/oder Biologika ein, davon $25 \%$ eine Kombination von konventionellen DMARDS und Biologika. Darüber hinaus erfolgte eine Behandlung mit Kortikosteroiden bei $71 \%$ der Patientinnen und Patienten mit TA.

Insgesamt wurden 152 Untersuchungstermine durch die 52 Patientinnen und $\mathrm{Pa}$ tienten wahrgenommen (median 3 [2-6] Untersuchungstermine/Patient). Bei 27 Patientinnen und Patienten erfolgte eine intensivierte Behandlung vor 36 Untersuchungen. Keine Veränderung der Behandlung erfolgte vor 21 Untersuchungsintervallen von 21 Patientinnen und Patienten und eine verringerte Behandlung erfolgte vor 32 Untersuchungsterminen von $24 \mathrm{~Pa}$ tientinnen und Patienten.
Wenn die Behandlung erhöht wurde, gab es eine signifikante Verringerung der Krankheitsaktivität, feststellbar anhand der bildgebenden, klinischen und entzündlichen Markern (jeweils $\mathrm{p} \leq 0,01$ ). Wenn die Behandlung unverändert blieb, veränderte sich auch nicht die Krankheitsaktivität über die 6-Monats-Intervalle. Wurde die Behandlung, das heißt die Einnahme von Kortikosteroiden und anderer Medikamente reduziert, verschlechterte sich der vaskuläre PET-Aktivitäts-Score signifikant $(p=0,02)$, die Marker der klinischen und serologischen Aktivität änderten sich hingegen nicht signifikant. Die spezifische GCA-Behandlung mit Tocilizumab und die Therapie der TA mit dem TNF-alpha-Inhibitor Infliximab bei einer Auswahl von Patienten, führten zu einer signifikanten Verbesserung der vaskulären Aktivität in der FDG-PETBildgebung sowie der klinischen Bewertung der Krankheitsaktivität, aber nur selten wurde eine klinische Remission und ein normales PET erreicht.

\section{FAZIT}

Zusätzlich zu klinischen und serologischen Bewertungen kann mit der Gefäßbildgebung die Krankheitsaktivität bei einer Entzündung großer Gefäße kontrolliert werden. Nach Auffassung des Autorenteams sollte das Verfahren in randomisierten klinischen Studien als Studienendpunkt getestet werden.

Richard Kessing, Zeiskam 